Research Paper

\title{
Diagnosis of Helicobacter pylori infection by invasive and noninvasive tests
}

\author{
Babak Pourakbari ${ }^{1}$, Mona Ghazi ${ }^{2}$, Shima Mahmoudi ${ }^{1}$, Setareh Mamishi ${ }^{1,3}$, Hossein \\ Azhdarkosh $^{4}$, Mehri Najafi ${ }^{5}$, Bahram Kazemi ${ }^{6}$, Ali Salavati ${ }^{7}$, Akbar Mirsalehian $^{8}$ \\ ${ }^{1}$ Pediatric Infectious Diseases Research Center, Tehran University of Medical Sciences, Tehran, Iran. \\ ${ }^{2}$ Department of Microbiology, School of Medicine, Shahid Beheshti University of Medical Sciences, \\ Tehran, Iran. \\ ${ }^{3}$ Department of Pediatric Infectious Diseases, School of Medicine, \\ Tehran University of Medical Sciences, Tehran, Iran. \\ ${ }^{4}$ Department of Internal Medicine, School of Medicine, \\ Tehran University of Medical Sciences, Tehran, Iran. \\ ${ }^{5}$ Department of Pediatric Gastroenterology, School of Medicine, \\ Tehran University of Medical Sciences, Tehran, Iran. \\ ${ }^{6}$ Cellular and Molecular Biology Research Center, \\ Shahid Beheshti University of Medical Sciences, Tehran, Iran. \\ ${ }^{7}$ Department of Epidemiology and Biostatistics, School of Public Health, \\ Tehran University of Medical Sciences, Tehran, Iran. \\ ${ }^{8}$ Department of Microbiology, School of Medicine, Tehran University of Medical Sciences, Tehran, Iran.
}

Submitted: November 2; Approved: July 23, 2012.

\begin{abstract}
Although several invasive and noninvasive tests have been developed for the diagnosis of Helicobacter pylori infection, all of the tests have their limitations. We conducted a study to investigate and compare the suitability of rapid urease test (RUT), serology, histopathology and stool antigen tests with polymerase chain reaction (PCR) for detection of $H$. pylori, and correlate the diagnostic methods with PCR. Eighty nine patients (61 adults, 28 children) referred to the Firoozgar Hospital and Children Medical Center Hospital for diagnostic upper gastrointestinal endoscopy entered to the study and noninvasive tests such as immunoassay for serological antibodies against $H$. pylori and detection of its antigen in feces were measured. The biopsies were utilized for histological examination, RUT and PCR. The H. pylori statuses were evaluated by the positivity of ureC PCR in biopsy specimens and 53 subjects had $H$. pylori positive result. Histopathology showed high overall performance in adults and children with sensitivity and specificity $100 \%$ and $90 \%$, respectively. Sensitivity, specificity, and accuracy for stool antigen test were $87.8 \%, 75 \%$ and $82 \%$, respectively. Correlation of RUT, serology $(\operatorname{IgG})$, histopathology and stool antigen tests with PCR were $0.82,0.32$, 0.91 and 0.63 , respectively. In conclusion, the RUT and histopathology are as accurate as the PCR of biopsy and stool antigen test can consider as appropriate noninvasive test for detection of $\mathrm{H}$. pylori infection.
\end{abstract}

Key words: Helicobacter pylori, diagnosis, invasive and noninvasive tests.

\section{Introduction}

A variety of methods for the detection of $H$. pylori have been described shortly after the identification of this pathogen and they have been continually improved and ex- tended over time (Dzierzanowska-Fangrat et al., 2006). Diagnostic methods for $H$. pylori infection are usually classified as invasive and noninvasive. The invasive tests including histology, urease tests and culture, require upper

Send correspondence to A. Mirsalehian. Department of Medical Microbiology, School of Medicine, Tehran University of Medical Sciences, no. 100 Poursina St., Tehran, Iran. E-mail: mirsaleh@sina.tums.ac.ir. 
gastrointestinal endoscopy for obtaining the diagnostic sample. On the other hand, non-invasive methods include the urea breath test, serology and stool antigen test (Dzierzanowska-Fangrat et al., 2006).

Although several diagnostic tests are available for the detection of $H$. pylori infection, all of them have both advantages and disadvantages. To define the value or usefulness of a diagnostic test, each test has to be compared to a gold standard (Guarner et al., 2010).

The aim of this study was to determine the accuracy of some noninvasive diagnostic methods in comparison with the invasive gold standard (endoscopy with biopsy analyses) for the diagnosis of $H$. pylori in Iranian patients with different upper gastrointestinal disorder.

\section{Material and Methods}

\section{Study population}

Eighty nine patients (61 adults, 28 children) referred to the Firoozgar Hospital and Children Medical Center (Teaching Hospitals affiliated to Tehran University of Medical Sciences) from May to October 2009 for diagnostic upper gastrointestinal endoscopy entered the study after providing written informed consent.

In all the patients, noninvasive tests such as immunoassay for serological antibodies against $H$. pylori and detection of its antigen in feces were measured. The biopsies were utilized for histological examination, rapid urease test (RUT) and polymerase chain reaction (PCR) of ureC.

\section{Definition of $H$. pylori status}

A positive $H$. pylori status was defined as positive results of PCR. A negative $H$. pylori status was confirmed when all invasive tests performed gave concordant negative results.

\section{Polymerase chain reaction}

The ureC ( $g \operatorname{lm} M)$ gene consider as a highly conserved and very sensitive and specific for the detection of $H$. pylori in gastric biopsy specimens (Bamford et al., 1998; Lu et al., 1999). The specimens used for PCR were gastric biopsy samples from patients who had undergone endoscopy. DNA was extracted by using a Bioneer AccuPrep extraction kit (Korea), according to the manufacturer's instructions. All PCR reagents were purchased from MBI Fermentas (Lithuania). PCR products were analyzed by gel electrophoresis using 1\% (w/v) UlteraPure agarose (invitrogen New Zealand), stained in $0.5 \mathrm{mg} / \mathrm{L}$ of ethidium bromide (Sigma-Aldrich, Australia), and examined by ultraviolet trans-illumination.

\section{Rapid Urease Test (RUT)}

A rapid urease test result was obtained by adding a biopsy specimen to a urea broth $(\mathrm{NaCl}, \mathrm{KH} 2 \mathrm{PO} 4$, and $\mathrm{NaOH}$ ); the result of the test was considered positive if there was a change of urea broth color from yellow-gold to pink-red due to an increase in $\mathrm{pH}$ induced by $H$. pylori (Sabbi et al., 2005)

\section{Histopathology}

Gastric biopsy specimens were immersed in 10\% formalin and embedded in paraffin. Sections were stained by hematoxylin and eosin, and modified Giemsa (Nogueira et $a l ., 2001)$. The stained sections were randomly renumbered before examination by observer and were assessed separately without knowledge of previous results, or of the proportions of positive $v s$. negative cases.

\section{Stool antigen tests based on immunoassay}

An enzyme immunoassay (ASTRA, Italy) was used to detect $H$. pylori in the frozen stool. A diluted feces sample and a peroxidase conjugated to antibody were added to the wells and incubated forl hour at room temperature. A wash was performed to remove unbound material. The substrate was added and incubated for $10 \mathrm{~min}$ at room temperature. Color developed in the presence of bound enzyme. Stop solution was added and the results were interpreted by spectrophotometer.

\section{Serum ELISA}

Blood samples were centrifuged, and serum was stored at $-20{ }^{\circ} \mathrm{C}$ for later analysis. ELISA (Monobind, USA) was used to detect $H$. pylori antibodies. Briefly, diluted serum samples were added to the coated wells with biotinylated conjugate solution and incubated before addition of a peroxidase-bound secondary immunoglobulin, incubation, and finally addition of a substrate showing $H$. pylori status.

\section{Statistics}

All analyses were performed using the user-written modules Diagt in STATA (release 10; StataCorp LP, College station, TX, USA). Sensitivity and specificity were combined into a single parameter, positive and negative predictive values, the likelihood ratio (LR): the odds (likelihood) of being infected if the test result was positive (LR+) and the odds of being infected if the test result is negative (LR-). Additionally, the accuracy was calculated as well as the corresponding $95 \%$ confidence intervals for all tests.

\section{Results}

Twenty eight children (12 males), aged $<18$ (mean $9.9 \pm 2.6 \mathrm{SD}$ years) and 61 adults (25 males), aged 19 to 81 years (mean $44.7 \pm 18.7$ SD years) were included in this study (Table 1). PCR products of the anticipated size (294 bp) were obtained from biopsy specimens of 49/89 $(55 \%)$ patients (in adults $32 / 61(52 \%)$, in children $17 / 28$ $61 \%)$ ) included in the study. 
Table 1 - Age and sex distribution of patients.

\begin{tabular}{lcccc}
\hline & No. of cases & \multicolumn{2}{c}{ Age (year) } & \multirow{2}{*}{ Sex Male/Female } \\
\cline { 3 - 4 } & & Mean \pm SD & Min-Max & \\
\hline Children $(<18)$ & 28 & $9.9 \pm 2.6$ & $6-16$ & $12 / 16$ \\
Adults & 61 & $44.7 \pm 18.7$ & $19-81$ & $25 / 36$ \\
Total & 89 & $33.7 \pm 22.4$ & $6-81$ & $37 / 52$ \\
\hline
\end{tabular}

The performance of various diagnostic techniques in all patients is shown in Table 2. Histopathology showed high overall performance, sensitivity $100 \%$ specificity $90 \%$ in both groups, RUT showed sensitivity $100 \%$ and $94 \%$ in children and adults, enzyme-linked immunosorbent assay (ELISA)-IgG assays showed low sensitivity (29\%) and high specificity (91\%) in children. In adult sensitivity and specificity was $62 \%$ and $80 \%$, respectively. IgA and IgM showed low performance and when PCR was positive these antibodies were found negative in all cases except 5 cases of children that IgA antibodies were positive. Stool antigen test accuracy, sensitivity, specificity, LR+ and LR- in the adult group were $91 \%, 79 \%, 4.38,0.12$ and 85 , respectively; whereas for the children group these values were $82 \%, 63 \%, 2.26,0.28$ and 75 , respectively.

Correlation of RUT, serology (IgG), histopathology and stool antigen tests with PCR in children were 1, 0.17, 0.92 and 0.46 , respectively; moreover, correlation of 0.77 , $0.41,0.9$, and 0.7 were found in adults group, respectively.

\section{Discussion}

The array of tests that can be used for diagnosis of $H$. pylori infection is large, and it might be confusing to define which test can use particularly in children. In our study histopathology showed high overall performance with accuracy of $95 \%$. An LR+ of 11 for RUT obtained from analysis of children provides moderate evidence that children with $H$. pylori infection have a greater chance of being RUT positive, compared with adults with the infection by LR + of 5.44. High LR+ estimate and low LR- estimate in RUT and histopathology, suggesting that these tests have a potential role in ruling out or confirming $H$. pylori infection in children.
Except serology of serum, other tests revealed good correlation with PCR. In general, serologic assays have a lower sensitivity especially in children and are not reliable in this group. These tests cannot be used on their own for diagnosis of $H$. pylori infection or to monitor the success of therapy because the sensitivity and specificity for detection of antibodies (IgG, IgM or IgA) against H. pylori varies widely and validity of serology in children is not well established (van Doorn et al., 2001). ELISA-IgG tests of serum in children showed high specificity, but low sensitivity and high LR- values. These findings have significant clinical implications, since a negative test would not be reliable for ensuring the absence of $H$. pylori infection. Low sensitivity might be explained due to the weak or immature immune response observed in young children. The corresponding LR- ratios of IgA, (children, 0.78 and adults 1.04) suggest that infection cannot be excluded when test results are negative. We observed that the immunological response of anti-H. pylori $\operatorname{IgG}$ antibodies was greater than that of anti-H. pylori IgA and IgM antibodies. The lack of IgM seropositivity in samples supports the transient nature of infections (Pérez-Pérez et al., 2003).

It has been reported that detection of $H$. pylori antigens in stool is a suitable noninvasive method for clinical and epidemiologic studies (Koletzko, 2005; Pourakbari et al., 2011).

In our study, the performance of stool antigen test in adult patients was excellent, with a sensitivity, specificity, and accuracy of $91 \%, 79 \%$ and $85 \%$, respectively. Lower efficiency of this test was found in children group. False positives or low specificity of stool antigen test can be explained by several mechanisms including the occurrence of transient H. pylori infection (spontaneous clearance of the

Table 2 - Test performance with its corresponding 95\% confidence intervals for each diagnostic test.

\begin{tabular}{lccccccc}
\hline & $\begin{array}{c}\text { Sensitivity (\%) } \\
(95 \% \mathrm{CI})\end{array}$ & $\begin{array}{c}\text { Specificity (\%) } \\
(95 \% \mathrm{CI})\end{array}$ & $\begin{array}{c}\text { Positive predic- } \\
\text { tive value }(\%) \\
(95 \% \mathrm{CI})\end{array}$ & $\begin{array}{c}\text { Negative predic- } \\
\text { tive value }(\%) \\
(95 \% \mathrm{CI})\end{array}$ & $\begin{array}{c}\text { Positive Likeli- } \\
\text { hood ratio (\%) } \\
(95 \% \mathrm{CI})\end{array}$ & $\begin{array}{c}\text { Negative Likeli- } \\
\text { hood ratio (\%) } \\
(95 \% \mathrm{CI})\end{array}$ & $\begin{array}{c}\text { Test accuracy } \\
(\%)(95 \% \mathrm{CI})\end{array}$ \\
\hline RUT & $95.9(86-99)$ & $85(70-94)$ & $88.7(77-96)$ & $94.4(81-99)$ & $6.39(3-13.4)$ & $0.048(0.01-0.18)$ & $91(84-94)$ \\
Histopathology & $100(93-100)$ & $90(76-97)$ & $92.5(82-98)$ & $100(90-100)$ & $10(3.95-25.3)$ & 0 & $95(90-95)$ \\
IgG & $50(35-65)$ & $83.3(67-94)$ & $79.3(60-92)$ & $56.6(42-70)$ & $3(1.37-6.58)$ & $0.6(0.43-0.83)$ & $65(54-71)$ \\
IgM & 0 & $94.4(81-99)$ & 0 & $42.5(31-54)$ & 0 & $1.06(0.98-1.15)$ & $41(41-46)$ \\
IgA & $60.9(45.4-74.9)$ & $69.4(51.9-83.7)$ & $71.8(55.1-85)$ & $58.1(42.1-73)$ & $1.99(1.16-3.43)$ & $0.563(0.37-0.86)$ & $64.6(54-74)$ \\
Stool Ag Kit & $87.8(75-95)$ & $75(59-97)$ & $81.1(68-91)$ & $83.3(67-94)$ & $3.51(2-6)$ & $0.163(0.07-0.35)$ & $82(73-88)$ \\
\hline
\end{tabular}


infection) which has been reported as a common phenomenon in children (Leal et al., 2008). The exposure to antibiotic therapy results in the conversion of the bacillary form to a coccoid form of $H$. pylori that is the morphological manifestation of bacterial cell death without an infective capacity. Thus, after eradication treatment, the $H$. pylori stool antigen test might detect antigen protein profiles resulting from the degradation of the two different morphobiological forms of $H$. pylori (Kusters et al., 1997; Forné et al., 2000; Masoero et al., 2000; Kabir, 2001). In addition, the sensitivity of the stool antigen test might decrease when the samples were received by $2-3$ days delay. Therefore, it may be concluded that unfrozen stool specimens should be sent within 1 day to a laboratory; otherwise the sensitivity is considerably reduced (Gisbert and Pajares, 2004).

Our study demonstrated that the RUT and histopathology, while being as accurate as the PCR of biopsy, are more burdensome to perform. Commercial enzyme immunoassays to detect $H$. pylori antibodies in serum have a lower accuracy, particularly in younger children, and making clinical decisions based on the results of these tests cannot be recommended (Drumm et al., 2000). Stool antigen test can consider as a suitable noninvasive test for detection of H. pylori infection.

\section{Acknowledgments}

This study was supported by Tehran University of Medical Sciences, Tehran, Iran with project grant number (87-04-30-8027).

\section{References}

Bamford K, Lutton D, O’Loughlin B, Coulter W, Collins J (1998) Nested primers improve sensitivity in the detection of Helicobacter pylori by the polymerase chain reaction. J Infect 36:105-110.

Drumm B, Koletzko S, Oderda G (2000) Helicobacter pylori infection in children: A consensus statement. J Pediatr Gastroenterol Nutr 30:207-213.

Dzierzanowska-Fangrat K, Lehours P, Mégraud F, Dzierzanowska D (2006) Diagnosis of Helicobacter pylori infection. Helicobacter 11:6-13.

Forné M, Domínguez J, Fernández-Bañares F, Lite J, Esteve M, Galí N, Espinós JC, Quintana S, Viver JM, (2000) Accuracy of an enzyme immunoassay for the detection of Helicobacter pylori in stool specimens in the diagnosis of infection and posttreatment check-up. Am J Gastroenterol 95:2200-2205.
Gisbert JP, Pajares JM (2004) Stool antigen test for the diagnosis of Helicobacter pylori infection: A systematic review. Helicobacter 9:347-368.

Guarner J, Kalach N, Elitsur Y, Koletzko S (2010) Helicobacter pylori diagnostic tests in children: Review of the literature from 1999 to 2009. Eur J Pediatr 169:15-25.

Kabir S (2001) Detection of Helicobacter pylori in faeces by culture, PCR and enzyme immunoassay. J Med Microbiol 50:1021-1029.

Koletzko S (2005) Noninvasive diagnostic tests for Helicobacter pylori infection in children. Can J Gastroenterol 19:433-439.

Kusters J, Gerrits M, Van Strijp J, Vandenbroucke-Grauls C (1997) Coccoid forms of Helicobacter pylori are the morphologic manifestation of cell death. Infect Immun 65:3672-3679.

Leal YA, Flores LL, García-Cortés LB, Cedillo-Rivera R, Torres $\mathrm{J}$ (2008) Antibody-based detection tests for the diagnosis of Helicobacter pylori infection in children: A meta-analysis. PLoS One 3:e3751.

Lu JJ, Perng CL, Shyu RY, Chen CH, Lou Q, Chong SK, Lee CH (1999) Comparison of five PCR methods for detection of Helicobacter pylori DNA in gastric tissues. J Clin Microbiol 37:772-774.

Masoero G, Lombardo L, Della Monica P, Vicari S, Crocilla C, Duglio A, Pera A (2000) Discrepancy between Helicobacter pylori stool antigen assay and urea breath test in the detection of Helicobacter pylori infection. Dig Liver Dis 32:285290.

Nogueira C, Figueiredo C, Carneiro F, Gomes AT, Barreira R, Figueira P, Salgado C, Belo L, Peixoto A, Bravo JC, Bravo LE, Realpe JL, Plaisier AP, Quint WG, Ruiz B, Correa P, van Doorn LJ (2001) Helicobacter pylori genotypes may determine gastric histopathology. Am J Pathol 158:647-654.

Pérez-Pérez GI, Sack RB, Reid R, Santosham M, Croll J, Blaser MJ (2003) Transient and persistent Helicobacter pylori colonization in Native American children. J Clin Microbiol 41:2401-2407.

Pourakbari B, Mirsalehian A, Maleknejad P, Mamishi S, Azhdarkosh H, Daryani NE, Najafi M, Kazemi B, Paknejad M, Mahmoudi S, Bandehpour M, Ghazi M, Salavati A (2011) Evaluation of a new antigen for diagnosis of Helicobacter pylori infection in stool of adult and children. Helicobacter 16:42-46.

Sabbi T, De Angelis P, Colistro F, Dall'Oglio L, di Abriola GF, Castro M (2005) Efficacy of noninvasive tests in the diagnosis of Helicobacter pylori infection in pediatric patients. Arch Pediatr Adolesc Med 159:238.

van Doorn OJ, Bosman DK, van't Hoff BW, Taminiau JA, Fibo J, van der Ende A (2001) Helicobacter pylori stool antigen test: a reliable non-invasive test for the diagnosis of Helicobacter pylori infection in children. Eur J Gastroenterol Hepatol 13:1061-1065.

All the content of the journal, except where otherwise noted, is licensed under a Creative Commons License CC BY-NC. 